\title{
Breve revisión y análisis sobre COVID-19 y lengua
}

\section{Brief review and analysis on COVID-19 and tongue}

\author{
Lilly Esquivel-Pedraza, ${ }^{\star}$ Laura Fernández-Cuevas, ${ }^{\ddagger}$ Judit Domínguez-Cherit
}

\section{RESUMEN}

El uso de las redes sociales durante la pandemia por COVID-19 se ha incrementado, generando también un aumento en la infodemia. El campo relativo a las manifestaciones bucales en la infección por el virus SARS-CoV-2 no ha sido ajeno a la desinformación, por lo que el presente manuscrito pretende aportar breves detalles y un análisis sobre los aspectos concernientes a los hallazgos bucales descritos en este grupo de pacientes, en particular en relación con la disgeusia.

Palabras clave: Manifestaciones bucales, SARS-CoV-2, COVID-19, disgeusia.

\section{ABSTRACT}

The use of social networks during the COVID-19 pandemic has increased, also generating an increase in infodemic. Mouth diseases observed in SARS-CoV-2 virus infection has not been the exception to misinformation; thus, this manuscript aims to provide brief details and an analysis identifying aspects of concern in oral findings, focused on dysgeusia.

Keywords: Mouth diseases, SARS-CoV-2, COVID-19, dysgeusia.

\section{INTRODUCCIÓN}

Debido a las medidas de aislamiento social implementadas a consecuencia de la pandemia por COVID-19 se ha incrementado el uso de las redes sociales. Estas plataformas digitales han tenido un desempeño muy importante en la divulgación rápida de abundante información médica y científica relativa a la pandemia. ${ }^{1}$ Desafortunadamente, en éstas es frecuente la difusión de datos no confiables o falsos. Por esta razón, en los siguientes párrafos se detallan algunos aspectos referentes a las alteraciones observadas en la mucosa lingual ocasionadas por la infección por SARS-CoV-2 con base en la literatura médica.

\section{SARS-CoV-2 y lengua}

La accesibilidad a los medios digitales por cualquier persona favorece que se compartan artículos o textos sin base científica. Con frecuencia, profesionales

* Departamento de Dermatología. Instituto Nacional de Ciencias Médicas y Nutrición "Dr. Salvador Zubirán».

‡ Clínica de Patología Bucal del Centro Dermatológico «Dr. Ladislao de la Pascua». no familiarizados con el campo de la medicina bucal atribuyen, de forma incorrecta, diversos síntomas y lesiones bucales como hallazgos patognomónicos de COVID-19, siendo que éstos también pueden observarse en otras enfermedades de la boca, por lo que, obviamente, no deben considerarse como exclusivos de esta infección viral.

En el contexto de la pandemia causada por el virus SARS-CoV-2, las alteraciones del gusto, también denominadas disgeusias (en concreto la ageusia), han motivado que, en la actualidad, la comunidad científica centre su atención en la anatomía y fisiología linguales. ${ }^{2}$

Algunos autores han sugerido que mucho antes de que un paciente se infecte con SARS-CoV-2, ya existe en su epitelio lingual algún tipo de disfunción en los corpúsculos gustativos, lo cual es el responsable directo del desarrollo de estos síntomas sensoriales. ${ }^{3}$ La vía de entrada de dicho virus a la célula huésped depende de la fusión de éste con el receptor de la enzima convertidora de angiotensina 2 (ECA2), expresado con amplitud en

Citar como: Esquivel-Pedraza L, Fernández-Cuevas L, Domínguez-Cherit J. Breve revisión y análisis sobre COVID-19 y lengua. Rev Cent Dermatol Pascua. 2021; 30 (3): 139-140. https://dx.doi.org/10.35366/103827 
las células de este epitelio. Esto explica claramente la susceptibilidad de la lengua a esta infección.

Esto contrasta con los hallazgos observados a partir de estudios de secuenciación de ARN (scRNA) que demuestran la existencia de receptores para diferentes tipos de virus en células epiteliales no gustativas, en particular en las células basales de las papilas filiformes, observándose que tan sólo una pequeña proporción incluye a las células del epitelio gustativo tipo $\mathrm{III} ; 4$ es decir, aquellas células neuroepiteliales de los corpúsculos gustativos que se especializan en la transmisión de la percepción del gusto. ${ }^{2}$

Con base en lo anterior, algunos autores sugieren que la ageusia e hipogeusia observadas en la infección por el virus SARS-CoV-2 se transmiten vía epitelio olfatorio hacia el cerebro, sin afectar de forma directa al epitelio lingual. ${ }^{5,6}$

En pacientes con COVID-19 se han detectado alteraciones en el color, textura y grosor de la lengua, como palidez, presencia de fisuras y superficie suave y «depapilada», todas ellas características comunes de la atrofia lingual. Algunos investigadores señalan que en pacientes con síntomas leves de infección por COVID-19 se aprecia una superficie lingual muy eritematosa, a diferencia de las fisuras y la atrofia de los casos graves. ${ }^{7}$ Cabe mencionar que estos estudios carecen de una descripción sobre el tipo de papilas afectadas, y tampoco correlacionan estas manifestaciones bucales con los factores que los condicionan, como podría ser una disminución de la concentración de hemoglobina y la subsecuente anemia ${ }^{8,9}$ observadas en algunos casos graves de la infección..$^{10,11}$ En el mismo contexto, tampoco se ha considerado tener en cuenta como diagnóstico diferencial a la infección candidósica, común entre la población general, y agente frecuente de datos clínicos linguales semejantes a los especificados con anterioridad. ${ }^{12}$

También se han descrito otras manifestaciones bucales por COVID-19, tales como la inflamación, el eritema y la subsecuente ulceración de la mucosa bucal. No obstante, es común observar estos signos en diversas infecciones virales $u$ otras entidades, ${ }^{9}$ que deben ser, por lo tanto, consideradas dentro de los diagnósticos diferenciales. ${ }^{10}$

\section{CONCLUSIÓN}

En la actualidad, las alteraciones del gusto han sido descritas erróneamente como distintivas de la infección por SARS-CoV-2; sin embargo, se debe tener en cuenta que éstas también se pueden presentar en otros padecimientos bucales, con predominio de faringoamigdalitis virales y bacterianas. Lo anterior obliga a hacer énfasis en la necesaria formación de especialistas en Patología
Bucal, enfocados en el área clínica, con el propósito de limitar la difusión de información imprecisa e inclusive errónea, que perjudica al paciente. De igual manera, se debe considerar a la ageusia como parte de los datos clínicos de las diversas enfermedades sistémicas que cursan con ella, y no sólo a la infección por SARS-CoV-2.

\section{REFERENCIAS}

1. Chaccour C, Vilasanjuan R. Infodemia: ¿Cómo ha afectado la epidemia de desinformación a la respuesta frente a la COVID-19? Inst Salud Globa. 2020: 1-10.

2. Fuentes A, Fresno MJ, Santander H, Valenzuela S, Gutiérrez MF, Miralles R. Gustatory sensory perception: a review. Int J Odontostomat. 2010; 4: 161-168.

3. Vandenbeuch A, Anderson CB, Parnes J, Enjyoji K, Robson SC, Finger TE, Kinnamon SC. Role of the ectonucleotidase NTPDase2 in taste bud function. Proceed Nat Acad Sci. 2013; 110: 14789-14794.

4. Xu H, Zhong L, Deng J, Peng J, Dan H, Zeng X et al. High expression of ACE2 receptor of 2019-nCoV on the epithelial cells of oral mucosa. Int J Oral Sci. 2020; 12: 8.

5. Butowt R, von Bartheld CS. Anosmia in COVID-19: underlying mechanisms and assessment of an olfactory route to brain infection. Neuroscientist. 2021; 27: 582-603.

6. Wang Z, Zhou J, Marshall B, Rekaya R, Ye K, Liu HX. SARS-CoV-2 receptor ACE2 is enriched in a subpopulation of mouse tongue epithelial cells in nongustatory papillae but not in taste buds or embryonic oral epithelium. ACS Pharmacol Transl Sci. 2020; 3: 749-758.

7. Pang W, Zhang D, Zhang J, Li N, Zheng W, Wang H et al. Tongue features of patients with coronavirus disease 2019: a retrospective cross-sectional study. Integrative Med Res. 2020; 9: 100493.

8. Wu YC, Wang YP, Chang JY, Cheng SJ, Chen HM, Sun A. Oral manifestations and blood profile in patients with iron deficiency anemia. J Formos Med Assoc. 2014; 113: 83-87.

9. Wu YC, Wu YH, Wang YP, Chang JY, Chen HM, Sun A. Hematinic deficiencies and anemia statuses in recurrent aphthous stomatitis patients with or without atrophic glossitis. J Formos Med Assoc. 2016; 115: 1061-1068.

10. Derruau S, Bouchet J, Nassif A, Baudet A, Yasukawa K, Lorimier S et al. COVID-19 and Dentistry in 72 questions: an overview of the literature. J Clin Med. 2021; 10: 779.

11. Huang N, Pérez P, Kato T, Mikami Y, Okuda K, Gilmore RC et al. SARS-CoV-2 infection of the oral cavity and saliva. Nat Med. 2021; 27: 892-903.

12. Terai H, Fukui N, Kasuya S, Hashiguchi N, Ueno T. Clinical features of partial atrophic tongue associated with Candida. Int J Dent Oral Sci. 2016; 3: 177-180.

\author{
Correspondencia: \\ Laura Fernández Cuevas \\ Hospital Médica Sur \\ Puente de Piedra Núm. 150, T1-317, \\ Col. Toriello Guerra, 14050 \\ Alcaldía Tlalpan, Ciudad de México, México. \\ Tel: 55-1917-8934 \\ E-mail: laurafercuervas@yahoo.com
}

